\title{
Vacancy-driven magnetocaloric effect in Prussian blue analogues
}

\author{
Marco Evangelisti ${ }^{\mathrm{a}, *}$, Espérança Manuel ${ }^{\mathrm{a}}$, Marco Affronte ${ }^{\mathrm{a}, \mathrm{b}}$, \\ Masashi Okubo ${ }^{\mathrm{c},}{ }^{\dagger}$, Cyrille Train $^{\mathrm{c}}$, Michel Verdaguer ${ }^{\mathrm{c}}$ \\ ${ }^{a}$ National Research Center on "nanoStructures and bioSystems at Surfaces" $\left(S^{3}\right)$, \\ INFM-CNR, 41100 Modena, Italy \\ b Dipartimento di Fisica, Università di Modena e Reggio Emilia, 41100 Modena, \\ Italy \\ ${ }^{\mathrm{c}}$ Chimie Inorganique et Matériaux Moléculaires, Unité CNRS 7071, Université \\ Pierre et Marie Curie, 75252 Paris, France
}

\begin{abstract}
We experimentally show that the magnetocaloric properties of molecule-based Prussian blue analogues can be adjusted by controlling during the synthesis the amount of intrinsic vacancies. For $\mathrm{Cs}_{x} \mathrm{Ni}_{4}^{I I}\left[\mathrm{Cr}^{I I I}(\mathrm{CN})_{6}\right]_{(8+x) / 3}$, we find indeed that the ferromagnetic phase transition induces significantly large magnetic entropy changes, whose maxima shift from $\sim 68 \mathrm{~K}$ to $\sim 95 \mathrm{~K}$ by varying the number of $\left[\mathrm{Cr}^{I I I}(\mathrm{CN})_{6}\right]^{3-}$ vacancies, offering an unique tunability of the magnetocaloric effect in this complex.
\end{abstract}

Key words: Magnetocaloric effect; Prussian blue analogues; Long-range

ferromagnetic order

PACS: 75.30.Sg; 75.40.Cx

Magnetic ordering phenomena are efficiently exploited to enhance the magnetocaloric effect (MCE) of magnetic materials [1]. This is possible because the response to the application or removal of magnetic fields is indeed maximized near the ordering temperature. In the search of suitable materials for magneto-cooling applications, however, one may need to adjust the ordering temperature to make optimum use of the magnetocaloric properties of a given material. For conventional materials, such as lanthanide compounds and alloys, it is common practice, in this respect, to partly substitute one constituting element for another one [1]. Here we show that a similar but different strategy can be employed as well in Prussian blue analogues (PBA),

\footnotetext{
* Corresponding author. Tel.: + 39-059-2055313.

Email address: evange@unimore.it (Marco Evangelisti).
} 


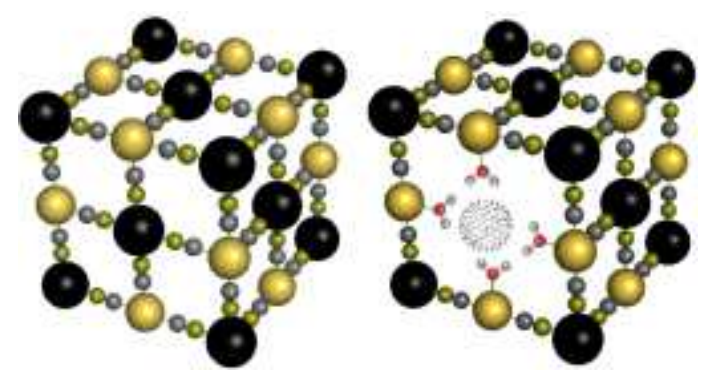

Fig. 1. Sketch of Ni-Cr PBA without any vacancy (left) and with a $\left[\mathrm{Cr}^{I I I}(\mathrm{CN})_{6}\right]^{3-}$ vacancy, shown as a large bright sphere, coordinated by bound water molecules (right). Black spheres represent $\mathrm{Cr}^{I I I}\left(S_{\mathrm{Cr}}=3 / 2\right)$, whereas lighter-colored spheres represent $\mathrm{Ni}^{I I}\left(\operatorname{spin} S_{\mathrm{Ni}}=1\right)$, and small circles denote the cyano-bridge that ensures the exchange coupling between the metallic centers.

which were recently investigated for their magnetocaloric properties [2]. The field of magnetocaloric research on molecule-based materials, such as PBA, is relatively young, although it has already shown promising potentialities [3].

The Prussian blue analogues here reported have the idealized formula $\mathrm{Cs}_{x} \mathrm{Ni}_{4}^{I I}\left[\mathrm{Cr}^{I I I}(\mathrm{CN})_{6}\right]_{(8+x) / 3}$, and the conventional unit cell is depicted in Fig. 1. Depending on the value of $x$, the presence of the intrinsic $\left[\mathrm{Cr}^{I I I}(\mathrm{CN})_{6}\right]^{3-}$ vacancies and their amount per cell is easily seen, as exemplified in the Figure. The non-stoichiometry is known to be essential for the observation of peculiar phenomena in this class of materials, such as photomagnetism for Co-Fe PBA [4]. In what follows, we shall focus on two Ni-Cr PBA having $x=0$ and $x=4$, which we shortly denote hereafter as $\mathrm{NiCr}_{2 / 3}$ and $\mathrm{CsNiCr}$, respectively. The difference between the two resides in the ideally perfect stoichiometry of $\mathrm{CsNiCr}$, for which half of the tetrahedral interstitial sites are occupied by Cs cations which maintain charge neutrality, and in the absence of $\mathrm{Cs}$ in $\mathrm{NiCr}_{2 / 3}$ that results in the presence of vacancies. Both compounds are known to undergo a transition to a long-range ferromagnetic ordered state [5]. Further information on the structure together with a description of the method of synthesis can be found in Ref. [5]. Susceptibility and magnetization measurements were carried out in a commercial apparatus for the $0<H<7 \mathrm{~T}$ magnetic field range. All data were collected on powdered samples of the compounds.

For both compounds, Figure 2 shows the complex susceptibility collected with an ac-field $h_{a c}=10 \mathrm{G}$ at $f=1730 \mathrm{~Hz}$. For CsNiCr, the abrupt change of the in-phase susceptibility $\chi^{\prime}(T)$ at $T_{C} \simeq 90 \mathrm{~K}$ is ascribed to the transition to a ferromagnetically ordered state, which is also corroborated by recent specific heat experiments [2]. Figure 2 shows as well that fluctuations in the ordering process gives rise to an out-of-phase susceptibility $\chi^{\prime \prime}(T)$ signal. The complex susceptibility of $\mathrm{NiCr}_{2 / 3}$ follows qualitatively that of $\mathrm{CsNiCr}$ but at much lower temperatures with a break in $\chi^{\prime}(T)$ at about $60 \mathrm{~K}$, which is accompanied by a $\chi^{\prime \prime}(T)$ signal (Fig. 2). The difference between the susceptibilities of the two compounds can be easily explained within the frame of the molecular field 


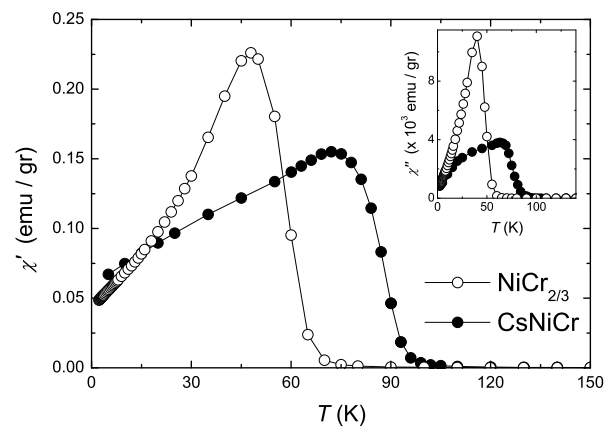

Fig. 2. Complex susceptibility collected at $f=1730 \mathrm{~Hz}$ and ac-field $h_{a c}=10 \mathrm{G}$, for CsNiCr and $\mathrm{NiCr}_{2 / 3}$, as labelled. Inset: out-of-phase component $\chi^{\prime \prime}(T)$.

theory for which the $T_{C}$ values of the present system can be expressed as

$$
\begin{aligned}
T_{C}= & \frac{2\left(z_{\mathrm{Ni}} z_{\mathrm{Cr}}\right)^{1 / 2}|J|}{3 k_{B}} \\
& \times\left[S_{\mathrm{Ni}}\left(S_{\mathrm{Ni}}+1\right) S_{\mathrm{Cr}}\left(S_{\mathrm{Cr}}+1\right)\right]^{1 / 2}
\end{aligned}
$$

where $z_{\mathrm{Ni}}$ and $z_{\mathrm{Cr}}$ are the numbers of nearest neighbour metal ions of the $\mathrm{Ni}^{I I}$ and $\mathrm{Cr}^{I I I}$ ions, respectively, and $J$ is the exchange coupling constant between the $\mathrm{Ni}^{I I}$ and $\mathrm{Cr}^{I I I}$ ions. The inclusion of $\left[\mathrm{Cr}^{I I I}(\mathrm{CN})_{6}\right]^{3-}$ vacancies accounts for the different $T_{C}$ 's, since it causes a change in the number of nearest neighbours. It is easy to show, indeed, that although $z_{\mathrm{Ni}}$ retains its value by switching from $\mathrm{CsNiCr}$ to $\mathrm{NiCr}_{2 / 3}$ (for which $z_{\mathrm{Ni}}=6$ ), the $z_{\mathrm{Cr}}$ values are 6 and 4 for $\mathrm{CsNiCr}$ and $\mathrm{NiCr}_{2 / 3}$, respectively. Given $S_{\mathrm{Ni}}=1, S_{\mathrm{Cr}}=2$ and assuming $T_{C}=90 \mathrm{~K}$ as for $\mathrm{CsNiCr}$, we obtain from Eq. (1) the exchange coupling whose estimate amounts to $J \simeq 6.5 \mathrm{~K}$, where the positive sign is set by the ferromagnetic nature of the ordered phase. By switching from CsNiCr to $\mathrm{NiCr}_{2 / 3}$, it follows, according to Eq. (1), a lower value for $T_{C}$ that amounts to $\sim 60 \mathrm{~K}$ for the latter, in good agreement with the susceptibility data (Fig. 2). In Prussian blue analogues, the dependence of $T_{C}$ on the number of neighbours is well-known, as was already reported for several derivatives including $\mathrm{Cs}_{x} \mathrm{Ni}_{4}^{I I}\left[\mathrm{Cr}^{I I I}(\mathrm{CN})_{6}\right]_{(8+x) / 3}$ as well $[5,6,7,8]$.

For a proper evaluation of the MCE of these compounds [9], we performed systematic magnetization $M(T, H)$ measurements as a function of temperature and field. Field-cooled $M(T, H)$ measurements for several applied-fields $H$ up to $7 \mathrm{~T}$ show spontaneous magnetization below the corresponding $T_{C}$ 's (Fig. 3 ). In an isothermal process of magnetization, the magnetic entropy change $\Delta S_{m}$ can be derived from Maxwell relations by integrating over the magnetic field 

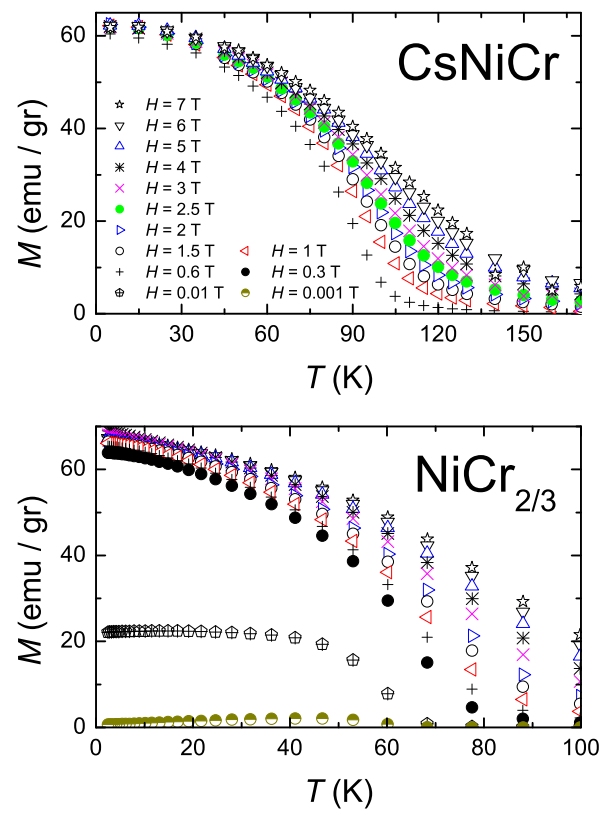

Fig. 3. Field-cooled $M(T)$ curves measured at different applied-fields for CsNiCr (top) and $\mathrm{NiCr}_{2 / 3}$ (bottom), as labelled in the top panel.

change $\Delta H=H_{f}-H_{i}$, that is:

$$
\Delta S_{m}(T)_{\Delta H}=\int_{H_{i}}^{H_{f}} \frac{\partial M(T, H)}{\partial T} \mathrm{~d} H .
$$

From the $M(H)$ data of Fig. 3, the obtained $\Delta S_{m}(T)$ for several $\Delta H$ values $[10]$ are depicted in Fig. 4. We note that the maximum change of the magnetic entropy upon application of a magnetic field, provides values that are similar for both compounds. Indeed, it can be seen that $-\Delta S_{m}$ increases by increasing $\Delta H$, reaching for $\Delta H=7 \mathrm{~T}$ the values of $6.6 \mathrm{~J} \mathrm{~kg}^{-1} \mathrm{~K}^{-1}$ and $6.9 \mathrm{~J} \mathrm{~kg}^{-1} \mathrm{~K}^{-1}$ for $\mathrm{CsNiCr}$ and $\mathrm{NiCr}_{2 / 3}$, respectively. However, since these changes are associated with the mechanism of magnetic ordering [2], it turns out that they take place at well-separated temperatures: $\sim 95 \mathrm{~K}$ and $\sim 68 \mathrm{~K}$, for $\mathrm{CsNiCr}$ and $\mathrm{NiCr}_{2 / 3}$, respectively (Fig. 4). Concluding, the possibility of controlling during the synthesis the number of vacancies, provides an excellent opportunity to shift the MCE of such complexes.

This work is partially funded by the Italian MIUR under FIRB project No. RBNE01YLKN and by the EC-Network of Excellence "MAGMANet" (No. 515767). E.M. acknowledges support from the EC-Marie Curie network "QuEMolNa" (No. MRTN-CT-2003-504880). M.O. was supported by the Japan Society for the Promotion of Science for Young Scientists. 

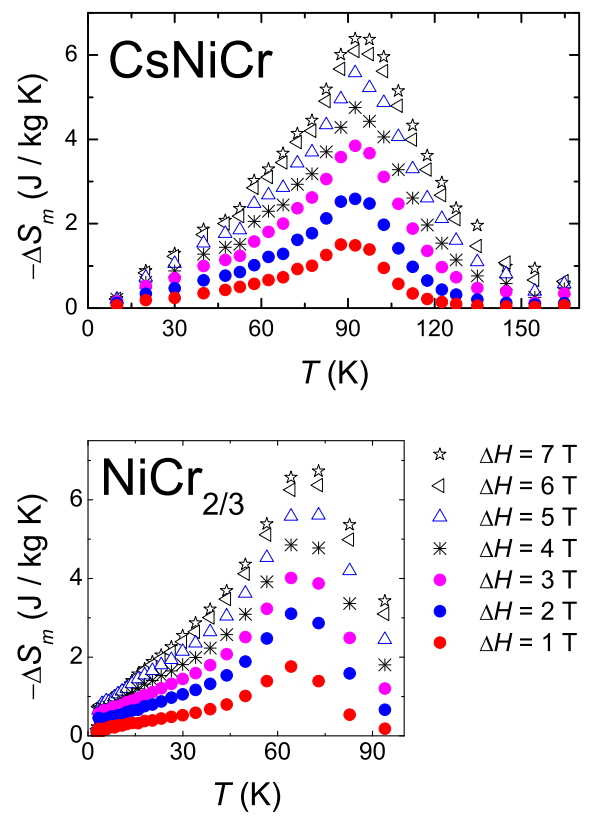

Fig. 4. Magnetic entropy change $\Delta S_{m}(T)$ as obtained from $M(T, H)$ data of Fig. 3 for $\mathrm{CsNiCr}$ (top) and $\mathrm{NiCr}_{2 / 3}$ (bottom), for several field changes $\Delta H$, as labelled.

\section{References}

[†] Present address: National Institute of Advanced Industrial Science and Technology (AIST), Umezono 1-1-1, Tsukuba, Ibaraki 305-8578, Japan.

[1] See, e.g., K.A. Gschneidner Jr., V.K. Pecharsky, A.O. Tsokol, Rep. Prog. Phys. 68 (2005) 1479, and references therein.

[2] E. Manuel, M. Evangelisti, M. Affronte, M. Okubo, C. Train, M. Verdaguer, Phys. Rev. B 73 (2006) 172406.

[3] For a recent review, see, e.g., M. Evangelisti, F. Luis, L.J. de Jongh, M. Affronte, J. Mater. Chem. (to be published), also cond-mat/0603368, and references therein.

[4] T. Kawamoto, Y. Asai, S. Abe, Phys. Rev. Lett. 86 (2001) 348.

[5] V. Gadet, T. Mallah, I. Castro, M. Verdaguer, J. Am. Chem. Soc. 114 (1992) 9213.

[6] W.D. Griebler and D. Babel, Z. Naturforsch. B 87 (1982) 832.

[7] M. Verdaguer, T. Mallah, V. Gadet, I. Castro, C. Hélary, S. Thiébaut, P. Veillet, Conf. Coord. Chem. 14 (1993) 19.

[8] S. Ohkoshi, O. Sato, T. Iyoda, A. Fujishima, K. Hashimoto, Inorg. Chem. 36 (1997) 268; S. Ohkoshi, T. Iyoda, A. Fujishima, K. Hashimoto, Phys. Rev. B 56 
(1997) 11642; S.-i. Ohkoshi and K. Hashimoto, Chem. Phys. Lett. 314 (1999) 210.

[9] V.K. Pecharsky and K.A. Gschneidner Jr., J. Appl. Phys. 86 (1999) 565.

[10] For practical reasons, the measurements at the lowest applied field were carried out for $H_{i}=10^{-3} \mathrm{~T}$, which in our calculations was approximated to zeroapplied-field. 